\title{
The Complexity of Hybrid Logics over Equivalence Relations
}

\author{
Martin Mundhenk • Thomas Schneider
}

Published online: 22 April 2009

(C) Springer Science+Business Media B.V. 2009

\begin{abstract}
This paper examines and classifies the computational complexity of model checking and satisfiability for hybrid logics over frames with equivalence relations. The considered languages contain all possible combinations of the downarrow binder, the existential binder, the satisfaction operator, and the global modality, ranging from the minimal hybrid language to very expressive languages. For model checking, we separate polynomial-time solvable from PSPACE-complete cases, and for satisfiability, we exhibit cases complete for NP, PSPACE, NEXPTIME, and even N2EXPTIME. Our analysis includes the versions of all these languages without atomic propositions, and also complete frames.
\end{abstract}

Keywords Hybrid logic · Downarrow operator - Satisfiability · Model checking

\section{Introduction}

Hybrid logics are powerful and well-behaved extensions of modal logic. However, their expressive power often claims a high price in terms of computational costs: Satisfiability for the language with the "downarrow binder" $\downarrow$ is undecidable Areces et al. (1999). ${ }^{1}$ Facing this drawback, it is natural to ask for restrictions under which

\footnotetext{
${ }^{1}$ The original proof appears in Blackburn and Seligman (1995), but uses four modalities. Goranko's proof in Goranko (1996) uses the global modality.
}

\section{Mundhenk ( $\varangle)$}

Institut für Informatik, Friedrich-Schiller-Universität, Jena, Germany

e-mail:martin.mundhenk@uni-jena.de

T. Schneider

School of Computer Science, University of Manchester, Manchester, UK

e-mail: schneider@cs.man.ac.uk 
decidability can be restored. One way is to restrict the syntax, for instance by disallowing certain combinations of $\downarrow$ and the $\square$ modality, which was examined in ten Cate and Franceschet (2005). Another way is to restrict the semantics by considering specific frame classes over which $\downarrow$ is not as expressive as over the class of all frames. A successful "taming" (i.e., decidability for satisfiability) of the $\downarrow$ language has been established for frames of bounded width in ten Cate and Franceschet (2005), and for transitive and complete frames in Mundhenk et al. (2005). Furthermore, over linear frames, where $\downarrow$ alone is useless, decidability has been shown for extensions of the $\downarrow$ language in Franceschet et al (2003).

Apparently, highly expressive hybrid languages can be tamed by restricting the class of frames. We will consider very restricted frame classes and will show that those languages still have high and different levels of complexity over them.

The starting point for our considerations is the NEXPTIME-completeness result for satisfiability of the $\downarrow$ language over complete frames from Mundhenk et al. (2005). What happens if we enrich the language and allow for slightly more general frames? We consider hybrid languages with and without propositional variables (the latter called pure languages) and each possible combination of $\downarrow, \exists$ (a binder stronger than $\downarrow$ ), the satisfaction operator @, and the stronger "somewhere" modality E. For these languages, we examine model checking and satisfiability over complete frames and over frames whose accessibility relation is an equivalence relation (ER frames for short). The results cover a spectrum from polynomial time up to nondeterministic doubly exponential time and thus exhibit the lack of robustness of binder languages.

The model-checking part of this paper mainly consists of consequences or refinements of results from Franceschet and de Rijke (2005), where the complexity of model checking for hybrid languages over arbitrary frames has been classified into polynomial-time computable and polynomial-space complete cases. We obtain a similar dichotomy. The interesting point is that the complexity only depends on whether the language contains $\downarrow$. Essentially, the complexity of model checking is PSPACEcomplete if the language contains $\downarrow$, and otherwise it is in polynomial time (Franceschet and de Rijke 2005). Whether the language is pure, and whether we are restricted to ER frames or even complete frames, does not affect complexity.

The satisfiability part contains new and technically involved results for highly expressive binder languages. For pure languages, we obtain a similar dichotomy as for model checking. Satisfiability is PSPACE-complete if the language contains $\downarrow$, and otherwise it is NP-complete. The frame class (complete resp. ER) is irrelevant. For "non-pure" languages, again a similar dichotomy is obtained for complete frames only. Satisfiability over complete frames is NEXPTIME-complete if the language contains $\downarrow$, and otherwise it is NP-complete. Over ER frames, we obtain an interesting difference for the language with $\downarrow$ and $E$ (which is the language with full expressivity). For all other languages, satisfiability over ER frames has the same complexity as over complete frames. Adding $E$ to $\downarrow$ or $\exists$ causes a whole exponential jump from NEXPTIME-completeness to N2EXPTIME-completeness, and adding $\downarrow$ to $E$ causes a doubly exponential jump from NP-completeness to N2EXPTIME-completeness. As we will show, this jump is due to two circumstances. First, the logic with $\downarrow$ and E lacks the exponential-size model property with respect to ER frames. This is because this language is expressive enough to enforce models of doubly exponential size. Second, 


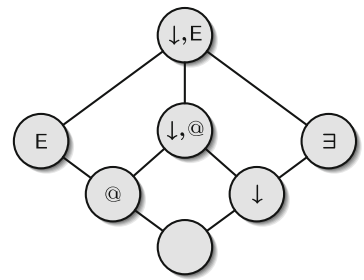

(a)

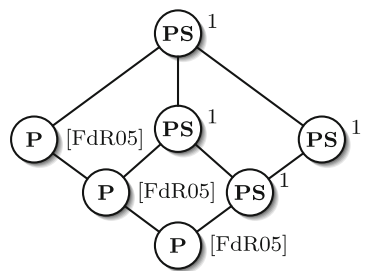

(b)

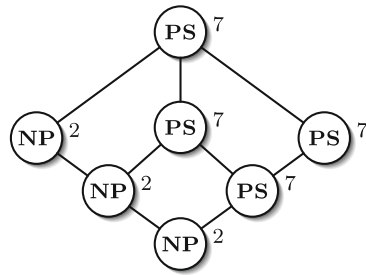

(c)

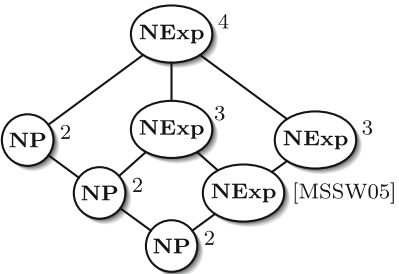

(d)

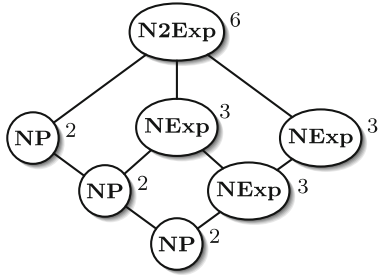

(e)

Fig. 1 A hierarchy of hybrid languages and an overview of their complexity. (a) hierarchy of all $\mathcal{H} \mathcal{L}(\cdot)$ (b) $[\mathcal{P}] \mathcal{H} \mathcal{L}(\cdot)$-compl-MC and $[\mathcal{P}] \mathcal{H} \mathcal{L}(\cdot)$-ER-MC (c) $\mathcal{P} \mathcal{H} \mathcal{L}(\cdot)$-compl-SAT and $\mathcal{P} \mathcal{H} \mathcal{L}(\cdot)$-ER-SAT (d) $\mathcal{H} \mathcal{L}(\cdot)$-compl-SAT (e) $\mathcal{H} \mathcal{L}(\cdot)$-ER-SAT

we can encode a N2EXPTIME-complete version of the the classical bounded tiling problem in these large models.

Our results are visualised in Fig. 1 b-e, where the nodes of the diagrams correspond to the languages given in part (a). These are obtained by analysing the interdefinabilities between the considered operators in Sect. 2, most of which are due to Blackburn and Seligman $(1995,1998)$. Part (b) of Fig. 1 shows the complexity of model checking, and parts (c)-(e) the complexity of satisfiability. The abbreviations $\mathcal{H} \mathcal{L}$ and $\mathcal{P} \mathcal{H} \mathcal{L}$ stand for the full and pure (without atomic propositions) language, respectively. ER and compl stand for ER frames resp. complete frames. The abbreviations in the nodes denote complexities: $\mathrm{P}$ for polynomial-time computable, and the rest for completeness with respect to NP, PSPACE, NEXPTIME, and N2EXPTIME. Each result is marked with the number of the respective theorem or a reference to its origin.

This paper is organised as follows. In Sect.2, we begin with basic concepts and notations of hybrid logic, complexity theory, and tilings. Sections 3 and 4 contain our results for model checking and satisfiability, respectively. We conclude in Sect. 5.

\section{Preliminaries}

\subsection{Hybrid Logic}

Hybrid languages are extensions of the modal language allowing for explicit references to states. Here we introduce the languages relevant for our work. The definitions and notations are taken from Areces et al. (1999, 2000). 
Syntax Let PROP be a countable set of propositional atoms, NOM be a countable set of nominals, SVAR be a countable set of state variables, and ATOM = PROP $\cup N O M \cup S V A R$. It is common practice to denote propositional atoms by $p, q, \ldots$, nominals by $i, j, \ldots$, and state variables by $x, y, \ldots$ The full hybrid language $\mathcal{H} \mathcal{L}(\downarrow, \exists, @, \mathrm{E})$ is the set of all formulae of the form $\varphi::=a|\neg \varphi| \varphi \wedge \varphi^{\prime} \mid$ $\diamond \varphi|\downarrow x . \varphi| \exists \varphi\left|@_{t} \varphi\right| \mathrm{E} \varphi$, where $a \in$ ATOM, $t \in$ NOM $\cup$ SVAR, and $x \in$ SVAR. We use the well-known abbreviations $\vee, \rightarrow, \leftrightarrow, \top$ ("true"), and $\perp$ ("false"), as well as $\square \varphi=\neg \diamond \neg \varphi, \forall \varphi=\neg \exists \neg \varphi$, and $\mathrm{A} \varphi=\neg \mathrm{E} \neg \varphi$. Whenever we leave $\downarrow$, @, or E out of the hybrid language, we omit the according symbol from $\mathcal{H} \mathcal{L}(\cdot)$.

A hybrid formula is called pure if it contains no propositional atoms; nominal-free if it contains no nominals; and a sentence if it contains no free state variables. (Free and bound are defined as usual; the only binding operators here are $\downarrow, \exists$.)

Semantics for $\mathcal{H} \mathcal{L}(\downarrow, \exists, @$, E) is defined in terms of Kripke models. A Kripke model is a triple $\mathcal{M}=(M, R, V)$, where $M$ is a nonempty set of states, $R \subseteq M \times M$ is a binary relation - the accessibility relation - , and $V:$ PROP $\rightarrow \mathfrak{P}(M)$ is a function - the valuation function. The structure $\mathcal{F}=(M, R)$ is called a frame.

A hybrid model is a Kripke model with the valuation function $V$ extended to PROP $\cup$ NOM, where for all $i \in \mathrm{NOM}, \# V(i)=1$. Whenever it is clear from the context, we will omit "hybrid" when referring to models. In order to evaluate $\downarrow$ - and $\exists$-formulae, an assignment $g:$ SVAR $\rightarrow M$ for $\mathcal{M}$ is necessary. Given an assignment $g$, a state variable $x$ and a state $m$, an $x$-variant $g_{m}^{x}$ of $g$ is defined by $g_{m}^{x}(x)=m$ and $g_{m}^{x}\left(x^{\prime}\right)=g\left(x^{\prime}\right)$ for all $x^{\prime} \neq x$. For any atom $a$, let $[V, g](a)=\{g(a)\}$ if $a \in$ SVAR, and $V(a)$, otherwise. Given a model $\mathcal{M}=(M, R, V)$, an assignment $g$, and a state $m \in M$, the satisfaction relation for hybrid formulae is defined by

$\begin{array}{ll}\mathcal{M}, g, m \Vdash a & \text { iff } m \in[V, g](a), a \in \text { ATOM, } \\ \mathcal{M}, g, m \Vdash \neg \varphi & \text { iff } \mathcal{M}, g, m \Vdash \varphi, \\ \mathcal{M}, g, m \Vdash \varphi \wedge \psi & \text { iff } \mathcal{M}, g, m \Vdash \varphi \& \mathcal{M}, g, m \Vdash \psi, \\ \mathcal{M}, g, m \Vdash \diamond \varphi & \text { iff for some } n \in M: m \operatorname{R} n \& \mathcal{M}, g, n \Vdash \varphi, \\ \mathcal{M}, g, m \Vdash \downarrow x . \varphi & \text { iff } \mathcal{M}, g_{m}^{x}, m \Vdash \varphi, \\ \mathcal{M}, g, m \Vdash \exists x . \varphi & \text { iff for some } n \in M: \mathcal{M}, g_{n}^{x}, m \Vdash \varphi, \\ \mathcal{M}, g, m \Vdash @{ }_{t} \varphi & \text { iff } \mathcal{M}, g, n \Vdash \varphi, \text { where }[V, g](t)=\{n\}, \\ \mathcal{M}, g, m \Vdash \mathrm{E} \varphi & \text { iff for some } n \in M: \mathcal{M}, g, n \Vdash \varphi .\end{array}$

A formula $\varphi$ is satisfiable if there exist a model $\mathcal{M}=(M, R, V)$, an assignment $g$ for $\mathcal{M}$, and a state $m \in M$, such that $\mathcal{M}, g, m \Vdash \varphi$.

The operators $\downarrow$ and $\exists$ are called binders; @ and $E$ are sometimes informally called jumping operators. There are certain dependencies between these four operators; most of them have been observed in Blackburn and Seligman $(1995,1998)$. First, $\downarrow$ can be expressed using $\exists: \downarrow x . \varphi$ is equivalent to $\exists x$. $(x \wedge \varphi)$. Second, $\exists$ can be expressed using $\downarrow$ and $\mathrm{E}: \exists x . \varphi$ is equivalent to $\downarrow y . \mathrm{E} \downarrow x$. $\mathrm{E}(y \wedge \varphi)$. Third, $\mathrm{E}$ can be expressed using $\exists$ and @: $\mathrm{E} \varphi$ is equivalent to $\exists x .\left(@_{x} \varphi\right)$. Fourth, @ can be expressed using E: @ ${ }_{x} \varphi$ is equivalent to $\mathrm{E}(x \wedge \varphi)$. In these formulae, $x$ and $y$ are state variables. Only in the last case can $x$ stand for a nominal, too. 
Because of these dependencies, arbitrary combinations of the operators $\downarrow, \exists$, @, E result in seven different hybrid languages: $\mathcal{H} \mathcal{L}, \mathcal{H} \mathcal{L}(@), \mathcal{H} \mathcal{L}(\mathrm{E}), \mathcal{H} \mathcal{L}(\downarrow), \mathcal{H} \mathcal{L}(\downarrow, @)$, $\mathcal{H} \mathcal{L}(\exists)$, and $\mathcal{H} \mathcal{L}(\downarrow, \mathrm{E})$. The inclusion hierarchy of these languages is given in Fig. 1a. All other combinations coincide with one of these languages. The pure fragment of $\mathcal{H} \mathcal{L}(X)$ is denoted by $\mathcal{P} \mathcal{H} \mathcal{L}(X)$.

Frame Classes; Satisfiability and Model Checking Problems Let $\mathcal{M}=(M, R, V)$ be a hybrid model with the underlying frame $\mathcal{F}=(M, R)$. If we require the accessibility relation to have certain properties, we restrict the class of relevant frames. Two frame classes are important for this paper. The class of complete frames is determined by the restriction $R=M \times M$, and the class of ER frames is the class of all frames with equivalence relations. In the latter case, call each equivalence class of $\mathcal{F}$ a cluster. A complete frame is an ER frame with one cluster only; obviously the E operator is not more expressive than $\diamond$ in this case.

For any hybrid language $\mathcal{H} \mathcal{L}(\cdot)$ and any frame class $\mathfrak{F}$, the satisfiability problem $\mathcal{H} \mathcal{L}(\cdot)-\mathfrak{F}$-SAT is defined as follows: Given a formula $\varphi \in \mathcal{H} \mathcal{L}(\cdot)$, do there exist a hybrid model $\mathcal{M}$ based on a frame from $\mathfrak{F}$, an assignment $g$ for $\mathcal{M}$, and a state $m \in M$ such that $\mathcal{M}, g, m \Vdash \varphi$ ? The model checking problem $\mathcal{H} \mathcal{L}(\cdot)-\mathfrak{F}-\mathrm{MC}$ is defined as follows: Given a formula $\varphi \in \mathcal{H} \mathcal{L}(\cdot)$, a hybrid model $\mathcal{M}$ based on a frame from $\mathfrak{F}$, and an assignment $g$ for $\mathcal{M}$, does $\mathcal{M}, g, m \Vdash \varphi$ hold for some state $m$ from $\mathcal{M}$ ? (If no binder is in the considered language, the assignment $g$ can be left out of either formulation.)

For example, the satisfiability problem over complete frames for the $\downarrow$ language is $\mathcal{H} \mathcal{L}(\downarrow)$-compl-SAT, while the model checking problem over ER frames for the $\exists$, @ language is denoted by $\mathcal{H} \mathcal{L}(\exists$, @ )-ER-MC.

It is straightforward to reduce $\mathcal{H} \mathcal{L}(X)$-compl-SAT to $\mathcal{H} \mathcal{L}(X)$-ER-SAT resp. $\mathcal{H} \mathcal{L}(X)$-compl-MC to $\mathcal{H} \mathcal{L}(X)$-ER-MC for arbitrary hybrid operator sets $X$. The reduction function defined by $f(\varphi)=\varphi \wedge \bigwedge_{i \in \operatorname{NOM}(\varphi)} \diamond i$ maps $\varphi$ to a formula that enforces that a satisfying ER model can be restricted to one cluster.

Bounded Model Properties A logic $\mathcal{H} \mathcal{L}(\cdot)$ is said to have the $f(n)$-size model property with respect to some class $\mathfrak{F}$ of frames, for some computable function $f: \mathbb{N} \rightarrow \mathbb{N}$, iff each formula $\varphi \in \mathcal{H} \mathcal{L}(\cdot)$ - $\mathfrak{F}$-SAT is satisfiable in a model from $\mathfrak{F}$ that has at most $f(|\varphi|)$ states. This property is important for proving upper complexity bounds of certain logics.

\subsection{Further Basic Concepts}

Complexity We refer to Papadimitriou (1994) for an introduction into complexity theory. In our classification, we use the complexity classes P and NP ((nondeterministic) polynomial time), PSPACE (polynomial space), NEXPTIME and N2EXPTIME (nondeterministic time $2^{\text {poly }(n)}$ and $2^{2^{\text {poly }(n)}}$, respectively). It is known that PSPACE is closed under nondeterminism, that is, PSPACE = NPSPACE. QSAT is a classical PSPACE-complete problem. It consists in determining whether a given Quantified Boolean Formula $(Q B F)$ is valid. QBF are first-order formulae of the form 
$\mathrm{Q}_{1} x_{1} \ldots \mathrm{Q}_{n} x_{n} \alpha\left(x_{1}, \ldots, x_{n}\right)$, where each $\mathrm{Q}_{i}$ is either $\exists$ or $\forall$, and $\alpha\left(x_{1}, \ldots, x_{n}\right)$ is a Boolean formula with only the $x_{i}$ as free variables.

Domino tiling problems are a helpful tool to establish lower complexity bounds for logics. A tile is a unit square, divided into four triangles by its diagonals. A tile type is a colouring of these four triangles and cannot be rotated. More formally, a tile type $t$ is a quadruple $t=(\operatorname{left}(t), \operatorname{right}(t), \operatorname{top}(t), \operatorname{bot}(t))$ of colours. Given a set $T$ of tile types, a $T$-tiling of the square with side length $n$ is a complete covering of that square with tiles having types from $T$, such that each point $(x, y)$ is covered by exactly one tile, adjacent tiles have the same colour at their common edges, and the outer border of the square is coloured white. Formally, a $T$-tiling of the $n \times n$ square is a function $\tau:\{0,1, \ldots, n-1\} \times\{0,1, \ldots, n-1\} \rightarrow T$ satisfying the following condition for all $(x, y) \in\{0,1, \ldots, n-1\} \times\{0,1, \ldots, n-1\}$.

$$
\begin{aligned}
\operatorname{right}(\tau(x, y)) & =\operatorname{left}(\tau(x+1, y)) \quad \& \quad \operatorname{top}(\tau(x, y))=\operatorname{bot}(\tau(x, y+1)) \\
\operatorname{bot}(\tau(x, 0)) & =\operatorname{top}(\tau(x, n-1))=\operatorname{left}(\tau(0, y)) \\
& =\operatorname{right}(\tau(n-1, y))=\text { white }
\end{aligned}
$$

The square tiling problem denotes the following question. Given a finite set $T$ of tile types and a string $1^{n}$ of $n$ consecutive $1 \mathrm{~s}$, is there a $T$-tiling of the square with side length $n$ ? This problem is NP-complete as was shown in Savelsbergh and van Emde Boas (1984). The proof technique used in Savelsbergh and van Emde Boas (1984) translates Turing machine computations into tilings and is very robust, such that simple variants of the square tiling problem can straightforwardly be shown to be complete for larger complexity classes. We will consider the following variant, which we call the $2^{2^{n}}$-Tiling problem. Given a finite set $T$ of tile types and a string $1^{n}$, is there a $T$-tiling of the $2^{2^{n}} \times 2^{2^{n}}$ square? This problem is N2EXPTIME-complete.

\section{Model Checking}

Franceschet and de Rijke Franceschet and de Rijke (2005) investigated model checking for hybrid logics with the $\downarrow$ and $\exists$ binders. Their hardness results hold for the pure nominal-free fragments of these languages. With a slight modification of their proof technique, it is possible to establish the same lower bound over complete frames.

Lemma $1 \mathcal{P H} \mathcal{H}(\downarrow)$-compl-MC is PSPACE-hard.

Proof We give a polynomial-time reduction from the standard PSPACE-complete problem QSAT. Consider an arbitrary instance $\psi=\mathrm{Q}_{1} x_{1} \ldots \mathrm{Q}_{n} x_{n} \alpha\left(x_{1}, \ldots, x_{n}\right)$ of QSAT. Let $\mathcal{M}=(M, R, V)$ consist of two states that form a complete frame, namely $M=\{1,2\}$ and $R=M \times M .^{2}$ From $\psi$ we construct a formula $h(\psi)=\downarrow t . \diamond(\downarrow$ $f .(\neg t \wedge \tau(\psi)))$ as follows. The first part ensures that $t$ is bound to one state of $M$ and

\footnotetext{
2 Notice that the size of $\mathcal{M}$ is independent of the size of the QSAT instance. Therefore, our proof even shows PSPACE-hardness of the expression complexity of $\mathcal{P} \mathcal{H} \mathcal{L}(\downarrow)$-compl-MC, i.e., this problem is PSPACE-hard even if the size of the model is considered fixed and only the size of the formula may vary.
} 
$f$ to the other. The formula $\tau(\psi)$ is obtained from $\psi$ by replacing all occurrences of variables $x_{i}$ by $\diamond\left(x_{i} \wedge t\right)$, all occurrences of $\exists x_{i}$ by $\diamond \downarrow x_{i}$, and all occurrences of $\forall x_{i}$ by $\square \downarrow x_{i}$. For example, the formula $\psi=\exists x_{1} \forall x_{2} \neg\left(x_{1} \vee\left(x_{2} \wedge \neg x_{1}\right)\right)$ is transformed to

$$
\begin{gathered}
h(\psi)=\downarrow t . \diamond\left(\downarrow f . \neg t \wedge \diamond\left(\downarrow x _ { 1 } \cdot \square \left(\downarrow x _ { 2 } \cdot \neg \left[\diamond ( x _ { 1 } \wedge t ) \vee \left(\diamond\left(x_{2} \wedge t\right)\right.\right.\right.\right.\right. \\
\left.\left.\left.\left.\left.\wedge \neg \diamond\left(x_{1} \wedge t\right)\right)\right]\right)\right)\right) .
\end{gathered}
$$

Then $\psi$ is true if and only if $\mathcal{M}, w \Vdash h(\psi)$ for an arbitrary $w \in M$.

A PSPACE upper bound for $\mathcal{H} \mathcal{L}(\downarrow, E)$-ER-MC follows from Franceschet and de Rijke (2005, Theorem 4.5). Together with Lemma 1 it combines to PSPACE-completeness of model-checking for hybrid languages as follows.

Theorem 1 Let $X$ be $\{\downarrow\},\{\downarrow, @\},\{\exists\}$, or $\{\downarrow, E\}$. Then $\mathcal{P} \mathcal{H} \mathcal{L}(X)$-compl-MC, $\mathcal{H} \mathcal{L}(X)$-compl-MC, $\mathcal{P} \mathcal{H} \mathcal{L}(X)$-ER-MC and $\mathcal{H} \mathcal{L}(X)$-ER-MC are PSPACE-complete.

Proof Containment in PSPACE follows from Franceschet and de Rijke (2005, Theorem 4.5), and hardness follows from Lemma 1.

\section{Satisfiability}

\subsection{The languages without binders}

We show NP-completeness of satisfiability for all pure and non-pure languages without binders, which is the same complexity as for modal logic over ER frames (Ladner 1977). The lower bound is almost trivial, and the upper bound is due to the $\mathcal{O}\left(n^{2}\right)$-size model property, which is established by a generalisation of the selection procedure given in Ladner (1977).

\section{Lemma $2 \mathcal{P} \mathcal{H} \mathcal{L}$-compl-SAT is NP-hard.}

Proof We reduce from the satisfiability problem SAT for propositional logic to $\mathcal{P} \mathcal{H} \mathcal{L}$-compl-SAT. Let $\varphi$ be a propositional formula with atomic propositions $p_{1}, \ldots$, $p_{n}$. The reduction function simply replaces each $p_{k}$ by a nominal $i_{k}$. Call the resulting hybrid formula $\varphi^{\prime}$. Clearly, if $\varphi$ is satisfiable, then there exists a satisfying assignment $\beta$ of all atomic propositions. A satisfying hybrid model for $\varphi^{\prime}$ consists of states $M=\{0,1\}$, the relation $R=M \times M$, and the valuation function defined by $V\left(i_{k}\right)=\left\{\beta\left(p_{k}\right)\right\}$. $(M, R)$ is a complete frame. Conversely, if $\varphi^{\prime}$ is satisfiable in a state $m$ of some hybrid model $\mathcal{M}=(M, R, V)$, then a satisfying assignment $\beta$ for $\varphi$ is obtained by setting $\beta\left(p_{k}\right)=1$ iff $V\left(i_{k}\right)=\{m\}$.

Lemma $3 \mathcal{H} \mathcal{L}(\mathrm{E})$ has the $\mathcal{O}\left(n^{2}\right)$-size model property with respect to ER frames.

Proof Let $\varphi \in \mathcal{H} \mathcal{L}(\mathrm{E})$-ER-SAT. Then there exists a hybrid model $\mathcal{M}=(M, R, V)$ and a state $m_{0,0} \in M$ such that $\mathcal{M}, m_{0,0} \Vdash \varphi$. Let $\mathrm{E} \psi_{1}, \ldots, \mathrm{E} \psi_{k}$ and $\diamond \vartheta_{1}, \ldots, \diamond \vartheta_{\ell}$ be all E- and $\diamond$-subformulae of $\varphi$. Now, for each $\mathrm{E} \psi_{i}$ that is satisfied at $m_{0,0}$, there is a state $m_{i, 0}$ satisfying $\psi_{i}$. For every other $\mathrm{E} \psi_{i}$ choose $m_{i, 0}=m_{0,0}$. Furthermore, for 
each of these $m_{i, 0}$ and each $\diamond \vartheta_{j}$ that is satisfied at $m_{i, 0}$, there is a state $m_{i, j}$ in the cluster of $m_{i, 0}$ satisfying $\vartheta_{j}$. For every other $\vartheta_{j}$, choose $m_{i, j}=m_{i, 0}$.

Now let $\mathcal{M}^{\prime}$ be the restriction of $\mathcal{M}$ to all $m_{i, j}$ with $i, j=0, \ldots, n$. This model clearly has at most $(n+1)^{2}$ states and contains $m_{0,0}$. The crucial fact $\mathcal{M}^{\prime}, m_{0,0} \Vdash \varphi$ follows from the claim that for each subformula $\psi$ of $\varphi$ and each $m_{i, j}: \mathcal{M}, m_{i, j} \Vdash \psi$ iff $\mathcal{M}^{\prime}, m_{i, j} \Vdash \psi$. This claim can be proven by a straightforward induction on $\psi$.

Let $\varphi$ be a formula from $\mathcal{H} \mathcal{L}(\mathrm{E})$ of length $n$. Due to the $\mathcal{O}\left(n^{2}\right)$-size model property, it suffices to guess a model of size $\mathcal{O}\left(n^{2}\right)$ and verify whether it satisfies $\varphi$. The last step can be done in time polynomial in $n$, due to Franceschet and de Rijke (2005, Theorem 4.3).

Both the Lemmas combine to NP-completeness of the satisfiability problem for all hybrid languages with $E$ as strongest operator over complete frames and $E R$ frames.

Theorem 2 Let $X$ be $\emptyset,\{@\}, \operatorname{or}\{\mathrm{E}\}$.Then $\mathcal{P H} \mathcal{L}(X)$-compl-SAT, $\mathcal{H} \mathcal{L}(X)$-compl-SAT, $\mathcal{P H} \mathcal{L}(X)$-ER-SAT and $\mathcal{H} \mathcal{L}(X)$-ER-SAT are NP-complete.

Proof NP-hardness follows from Lemma2. It remains to show containment in NP of $\mathcal{H} \mathcal{L}(\mathrm{E})$-ER-SAT. Let $\varphi$ be a formula from $\mathcal{H} \mathcal{L}(\mathrm{E})$ of length $n$. Due to the $\mathcal{O}\left(n^{2}\right)$ size model property (Lemma3), it suffices to guess a model of size $\mathcal{O}\left(n^{2}\right)$ and verify whether it satisfies $\varphi$. The last step can be done in time polynomial in $n$, due to Franceschet and de Rijke (2005, Theorem 4.3).

\subsection{The languages with binders and without $\mathrm{E}$}

For the remaining languages, the complexity of model checking for pure languages is lower than for the non-pure languages. We will deal with the pure languages in Sect. 4.4.

Next, we consider the languages $\mathcal{H} \mathcal{L}(\downarrow), \mathcal{H} \mathcal{L}(\downarrow$, @ ), and $\mathcal{H} \mathcal{L}(\exists)$ and show that satisfiability over complete frames and over ER frames is NEXPTIME-complete (Theorem 3). Using the hierarchy of the languages, it suffices to use that $\mathcal{H} \mathcal{L}(\downarrow)$-compl-SAT is NEXPTIME-hard (Mundhenk et al., 2005, Theorem 4), and that $\mathcal{H} \mathcal{L}(\downarrow$, @ )-ER-SAT and $\mathcal{H} \mathcal{L}(\exists)$-ER-SAT are in NEXPTIME (Lemmas 4 and 5).

Lemma $4 \mathcal{H} \mathcal{L}(\downarrow$, @ )-ER-SAT is in NEXPTIME.

Proof It suffices to reduce $\mathcal{H} \mathcal{L}(\downarrow$, @ )-ER-SAT to $\mathcal{H} \mathcal{L}(\downarrow)$-compl-SAT, which is in NEXPTIME (Mundhenk et al. 2005). This reduction will rely on two basic observations. First, it suffices to consider sentences only, because free state variables can be replaced by nominals without affecting satisfiability. Second, a satisfying ER model for an $\mathcal{H} \mathcal{L}(\downarrow, @)$ sentence $\varphi$ consists w.l.o.g. of not more clusters than there are nominals in $\varphi$ plus one.

To put the last observation more formally, let $\varphi$ be an $\mathcal{H} \mathcal{L}(\downarrow$, @) sentence with nominals $i_{1}, \ldots, i_{n}$. If $\varphi$ is satisfied in a state $m$ of a model $\mathcal{M}$, then $\varphi$ is satisfied in the restriction of $\mathcal{M}$ to the clusters that contain $m$ and all $V\left(i_{k}\right)$. This is so because other clusters are not accessible by means of $\diamond$ or @. 
Hence we can assume w.l.o.g. that a satisfying model for $\varphi$ consists of at most $n+1$ clusters. Clearly $n \leqslant|\varphi|$. Such a model can be transformed into a model consisting of one "new" cluster that is the union of all these "old" clusters. The old clusters can be distinguished by fresh atomic propositions $c_{0}, \ldots, c_{n}$, which help simulate $\diamond$ and @ using only $\diamond$. This simulation is captured by the following translation from $\mathcal{H} \mathcal{L}(\downarrow$, @ ) to $\mathcal{H} \mathcal{L}(\downarrow)$ using a fresh state variable $x$.

$$
\begin{array}{ll}
a^{t}=a, \quad \text { for } \quad a \in \mathrm{ATOM} & (\diamond \varphi)^{t}=\downarrow x . \diamond\left(\bigwedge_{k=0}^{n}\left(c_{k} \leftrightarrow \square\left(x \rightarrow c_{k}\right)\right) \wedge \varphi^{t}\right) \\
(\neg \varphi)^{t}=\neg \varphi^{t} & \left(@_{v} \varphi\right)^{t}=\diamond\left(v \wedge \varphi^{t}\right) \\
(\varphi \wedge \psi)^{t}=\varphi^{t} \wedge \psi^{t} & (\downarrow v \cdot \varphi)^{t}=\downarrow v \cdot \varphi^{t}
\end{array}
$$

With the help of the translation $(\cdot)^{t}$, we define the reduction function $f: \mathcal{H} \mathcal{L}(\downarrow$, @) $\rightarrow \mathcal{H} \mathcal{L}(\downarrow)$ by

$$
\begin{gathered}
f(\varphi)=\varphi^{t} \wedge c_{0} \wedge \square \bigvee_{k=0}^{n} c_{k} \wedge \square\left(i_{k} \rightarrow c_{k}\right) \wedge \bigwedge_{\substack{k, \ell=0, \ldots, n \\
k \neq \ell}}\left(\left(c_{k} \leftrightarrow c_{\ell}\right)\right. \\
\left.\vee \square\left(\left(c_{k} \rightarrow \neg c_{\ell}\right) \wedge\left(c_{\ell} \rightarrow \neg c_{k}\right)\right)\right),
\end{gathered}
$$

where the conjuncts after $\varphi^{t}$ express that $\varphi$ is satisfied in cluster 0 ; each state of the new cluster belongs to some old cluster; nominal $i_{k}$ is true in cluster $k$; and two clusters $k, \ell$ are either equal or disjoint. It remains to prove that $\varphi \in \mathcal{H} \mathcal{L}(\downarrow, @)$-ER-SAT if and only if $f(\varphi) \in \mathcal{H} \mathcal{L}(\downarrow)$-compl-SAT.

“ $\Rightarrow$ ”. Suppose $\varphi \in \mathcal{H} \mathcal{L}(\downarrow, @)$-ER-SAT. Then there exist a model $\mathcal{M}=(M, R, V)$, a state $m_{0} \in M$, and an assignment $g_{0}$ for $\mathcal{M}$ such that $\mathcal{M}, g_{0}, m_{0} \Vdash \varphi$. Without loss of generality, $\mathcal{M}$ has only those clusters that are determined by $m$ and all $V\left(i_{k}\right)$. Let $V\left(i_{k}\right)=m_{k}$, for $k=1, \ldots, n$. We construct a model $\mathcal{M}^{\triangleright}=\left(M^{\triangleright}, R^{\triangleright}, V^{\triangleright}\right)$, where $M^{\triangleright}=M, R=M^{\triangleright} \times M^{\triangleright}$, and define $V^{\triangleright}$ by $V^{\triangleright}(a)=V(a)$ for $a \in$ PROP $\cup$ NOM, and $V^{\triangleright}\left(c_{k}\right)=\left\{m \in M \mid m R m_{k}\right\}$ for $k=0, \ldots, n$. Furthermore, for each assignment $g$ for $\mathcal{M}$, define the assignment $g^{\triangleright}$ for $\mathcal{M}^{\triangleright}$ by $g^{\triangleright}(y)=y$ for each $y \neq x$, and $g^{\triangleright}(x)=m_{0}$.

We have to show that $\mathcal{M}^{\triangleright}, g_{0}^{\triangleright}, m_{0} \Vdash f(\varphi)$. It is immediately clear from the construction that the conjuncts following $\varphi^{t}$ in $f(\varphi)$ are satisfied in $m_{0}$ of $\mathcal{M}^{\triangleright}$ under $g_{0}^{\triangleright}$. The fact that $\mathcal{M}^{\triangleright}, g_{0}^{\triangleright}, m_{0} \Vdash \varphi^{t}$ is a consequence of the following claim.

Claim For each subformula $\psi$ of $\varphi$, for each state $m \in M$, and for each assignment $g$ for $\mathcal{M}$ :

$$
\mathcal{M}, g, m \Vdash \psi \quad \text { if and only if } \quad \mathcal{M}^{\triangleright}, g^{\triangleright}, m \Vdash \psi^{t} \text {. }
$$

Proof of Claim We proceed by induction on the structure of $\psi$. The atomic and Boolean cases follow immediately from the construction. The cases for @ and $\downarrow$ are straightforward. It remains to discuss the only interesting case $\psi=\diamond \vartheta$, which is done via the following chain of equivalent statements. 


$$
\begin{aligned}
& \mathcal{M}, g, m \Vdash \diamond \vartheta \\
& \Leftrightarrow \exists \ell \in M[m R \ell \& \mathcal{M}, g, \ell \Vdash \vartheta] \\
& \Leftrightarrow \exists \ell \in M\left[m R \ell \& \mathcal{M}^{\triangleright}, g^{\triangleright}, \ell \Vdash \vartheta^{t}\right] \\
& \Leftrightarrow \exists \ell \in M\left[m R \ell \& \mathcal{M}^{\triangleright},\left(g^{\triangleright}\right)_{m}^{x}, \ell \Vdash \vartheta^{t}\right] \\
& \Leftrightarrow \exists \ell \in M^{\triangleright}\left[\forall k \leqslant n\left(\ell \in V^{\triangleright}\left(c_{k}\right) \Leftrightarrow m \in V^{\triangleright}\left(c_{k}\right)\right)\right. \\
& \left.\& \mathcal{M}^{\triangleright},\left(g^{\triangleright}\right)_{m}^{x}, \ell \Vdash \vartheta^{t}\right] \\
& \Leftrightarrow \exists \ell \in M^{\triangleright}\left[\mathcal{M}^{\triangleright},\left(g^{\triangleright}\right)_{m}^{x}, \ell \Vdash \bigwedge_{k=0}^{n}\left(c_{k} \leftrightarrow \square\left(x \rightarrow c_{k}\right)\right)\right. \\
& \left.\& \mathcal{M}^{\triangleright},\left(g^{\triangleright}\right)_{m}^{x}, \ell \Vdash \vartheta^{t}\right] \\
& \Leftrightarrow \mathcal{M}^{\triangleright},\left(g^{\triangleright}\right)_{m}^{x}, m \Vdash \diamond\left(\bigwedge_{k=0}^{n}\left(c_{k} \leftrightarrow \square\left(x \rightarrow c_{k}\right)\right) \wedge \vartheta^{t}\right) \text { (satisfaction rules) } \\
& \Leftrightarrow \mathcal{M}^{\triangleright}, g^{\triangleright}, m \Vdash \downarrow x . \diamond\left(\bigwedge_{k=0}^{n}\left(c_{k} \leftrightarrow \square\left(x \rightarrow c_{k}\right)\right) \wedge \vartheta^{t}\right) \text { (satisfaction rules) } \\
& \Leftrightarrow \mathcal{M}^{\triangleright}, g^{\triangleright}, m \Vdash(\diamond \vartheta)^{t}
\end{aligned}
$$

“Æ”. $\quad$ Suppose $\varphi \in \mathcal{H} \mathcal{L}(\downarrow)$-compl-SAT. Then there exist a model $\mathcal{M}=(M, R, V)$, a state $m_{0} \in M$, and an assignment $g_{0}$ for $\mathcal{M}$ such that $\mathcal{M}, g_{0}, m_{0} \Vdash f(\varphi)$. Due to the conjuncts after $\varphi^{t}$ in $f(\varphi)$, the variables $c_{k}$ "almost partition" $M$ in the following sense. Let $\mathrm{Cl}_{k}=V\left(c_{k}\right)$. Then $m_{0} \in \mathrm{Cl}_{0}$; for each state $m \in M$ there is some $k \leqslant n$ with $m \in \mathrm{Cl}_{k} ; V\left(i_{k}\right) \subseteq \mathrm{Cl}_{k}$; and for two disjoint $k, \ell \leqslant n$, either $\mathrm{Cl}_{k}=\mathrm{Cl}_{\ell}$ or $\mathrm{Cl}_{k} \cap \mathrm{Cl}_{\ell}=\emptyset$.

Hence the following construction of a model $\mathcal{M}^{\triangleleft}=\left(M^{\triangleleft}, R^{\triangleleft}, V^{\triangleleft}\right)$ is correct. Let $M^{\triangleleft}=M, R^{\triangleleft}=\left\{\left(m, m^{\prime}\right) \mid \forall k \leqslant n\left(m \in \mathrm{Cl}_{k} \Leftrightarrow m^{\prime} \in \mathrm{Cl}_{k}\right)\right\}$, and $V^{\triangleleft}$ be the restriction of $V$ to (NOM $\cup$ PROP) $-\bigcup\left\{c_{k}\right\}$. Furthermore, for each assignment $g$ for $\mathcal{M}$, let $g^{\triangleleft}=g$, which is an assignment for $\mathcal{M}^{\triangleleft}$.

It remains to show that $\mathcal{M}^{\triangleleft}, g_{0}^{\triangleleft}, m_{0} \Vdash \varphi$, which is a consequence of $\mathcal{M}, g_{0}, m_{0} \Vdash$ $\varphi^{t}$ and the following claim.

Claim For each subformula $\psi$ of $\varphi$, for each state $m \in M$, and for each assignment $g$ for $\mathcal{M}$ :

$$
\mathcal{M}, g, m \Vdash \psi^{t} \quad \text { if and only if } \quad \mathcal{M}^{\triangleleft}, g^{\triangleleft}, m \Vdash \psi .
$$

Proof of Claim We proceed by induction on the structure of $\psi$. Again, the atomic and Boolean cases follow immediately from the construction, and the cases for @ and $\downarrow$ are straightforward. It remains to discuss the only interesting case $\psi=\diamond \vartheta$, which is done via the following chain of equivalent statements.

$$
\begin{aligned}
& \mathcal{M}, g, m \Vdash(\diamond \vartheta)^{t} \\
& \Leftrightarrow \mathcal{M}, g, m \Vdash \downarrow x . \diamond\left(\bigwedge_{k=0}^{n}\left(c_{k} \leftrightarrow \square\left(x \rightarrow c_{k}\right)\right) \wedge \vartheta^{t}\right) \\
& \Leftrightarrow \mathcal{M}, g_{m}^{x}, m \Vdash \diamond\left(\bigwedge_{k=0}^{n}\left(c_{k} \leftrightarrow \square\left(x \rightarrow c_{k}\right)\right) \wedge \vartheta^{t}\right) \quad \text { (satisfaction rules) } \\
& \Leftrightarrow \exists \ell \in M\left[\mathcal{M}, g_{m}^{x}, \ell \Vdash \bigwedge_{k=0}^{n}\left(c_{k} \leftrightarrow \square\left(x \rightarrow c_{k}\right)\right)\right. \\
& \left.\& \mathcal{M}, g_{m}^{x}, \ell \Vdash \vartheta^{t}\right] \\
& \Leftrightarrow \exists \ell \in M\left[\forall k \leqslant n\left(\ell \in \mathrm{Cl}_{k} \Leftrightarrow m \in \mathrm{Cl}_{k}\right)\right. \\
& \left.\& \mathcal{M}, g_{m}^{x}, \ell \Vdash \vartheta^{t}\right] \\
& \Leftrightarrow \exists \ell \in M^{\triangleleft}\left[m R^{\triangleleft} \ell \& \mathcal{M}, g_{m}^{x}, \ell \Vdash \vartheta^{t}\right] \\
& \Leftrightarrow \exists \ell \in M^{\triangleleft}\left[m R^{\triangleleft} \ell \& \mathcal{M}, g, \ell \Vdash \vartheta^{t}\right] \\
& \Leftrightarrow \exists \ell \in M^{\triangleleft}\left[m R^{\triangleleft} \ell \& \mathcal{M}^{\triangleleft}, g^{\triangleleft}, \ell \Vdash \vartheta\right] \\
& \Leftrightarrow \mathcal{M}^{\triangleleft}, g^{\triangleleft}, m \Vdash \diamond \vartheta \\
& \text { (satisfaction rules) } \\
& \text { (satisfaction rules) } \\
& \text { (construction of } M^{\triangleleft}, R^{\triangleleft} \text { ) } \\
& \text { (since } x \text { is bound in } \vartheta^{t} \text { ) } \\
& \text { (induction hypothesis) } \\
& \text { (satisfaction rules) }
\end{aligned}
$$

This ends the proof. 
Lemma $5 \mathcal{H} \mathcal{L}(\exists)$-ER-SAT is in NEXPTIME.

Proof The $\exists$ binder can bind state variables to states that are not accessible using $\diamond$. In this case, the bound variable evaluates to false. Therefore, if $\varphi \in \mathcal{H} \mathcal{L}(\exists)$-ER-SAT and $\mathcal{M}, g_{0}, m_{0} \Vdash \varphi$, we can modify $\mathcal{M}=(M, R, V)$ to a model $\mathcal{M}^{\prime}=\left(M^{\prime}, R^{\prime}, V^{\prime}\right)$, where $M^{\prime}$ consists of the states of the cluster $C$ with $m_{0} \in C$ plus one additional state $s \notin C$. $R^{\prime}$ is the restriction of $R$ to $M^{\prime}$, and $V^{\prime}(p)=V(p) \cap M^{\prime}$, for atomic propositions $p$. For nominals $i$, define $V^{\prime}(i)=V(i)$ if $V(i) \subseteq M^{\prime}$, and $V^{\prime}(i)=\{s\}$, otherwise. For each assignment $g$ for $\mathcal{M}$, the corresponding assignment $g^{\prime}$ for $\mathcal{M}$ is obtained from $g$ by binding all variables to $s$ that are bound to states outside of $C$ by $g$. It is straightforward that $\mathcal{M}, g, m \Vdash \psi$ if and only if $\mathcal{M}^{\prime}, g^{\prime}, m \Vdash \psi$, for any state $m \in C$, any assignment $g$ for $\mathcal{M}$, and any subformula $\psi$ of $\varphi$ (proof by induction). This implies $\mathcal{M}^{\prime},\left(g_{0}\right)^{\prime}, m_{0} \Vdash \varphi$.

Now, $\mathcal{M}^{\prime}$ is a model with two clusters only. Transforming $\mathcal{M}^{\prime}$ into an appropriate complete model as in the proof of Lemma 4 reduces $\mathcal{H} \mathcal{L}(\exists)$-ER-SAT to $\mathcal{H} \mathcal{L}(\downarrow)$-compl-SAT, which is in NEXPTIME (Mundhenk et al. 2005). From (Mundhenk et al., 2005, Theorem 4), Lemma 4 and Lemma 5 we obtain the complete characterisation of the satisfiability problems for hybrid logics with $\downarrow$ and without $\mathrm{E}$.

Theorem 3 Let $X$ be $\{\downarrow\}, \quad\{\exists\}$, or $\{\downarrow, @\}$. Then $\mathcal{H} \mathcal{L}(X)$-compl-SAT and $\mathcal{H} \mathcal{L}(X)$-ER-SAT are NEXPTIME-complete.

\subsection{The full language}

For complete frames, the E-operator does not add any expressive power to the language, since $\mathrm{E} \varphi$ is equivalent to $\diamond \varphi$. Therefore, the complexity of $\mathcal{H} \mathcal{L}(\downarrow, E)$-compl-SAT is the same as for $\mathcal{H} \mathcal{L}(\downarrow)$-compl-SAT (see Theorem 3 ).

\section{Theorem $4 \mathcal{H} \mathcal{L}(\downarrow, \mathrm{E})$-compl-SAT is NEXPTIME-complete.}

In contrast, the complexity of $\mathcal{H} \mathcal{L}(\downarrow, E)$-ER-SAT is one exponential level higher. Our results for the satisfiability of $\mathcal{H} \mathcal{L}(\downarrow$, @ ) and $\mathcal{H} \mathcal{L}(\exists)$ over ER frames rely on the fact that satisfying models with at most two clusters can be found for satisfiable formulas. This is not the case for $\mathcal{H} \mathcal{L}(\downarrow, E)$. We present a satisfiable formula that has no model below doubly exponential size. We will show that it is possible, but not quite straightforward, to enforce a tiling in such big models, which establishes N2EXPTIME-hardness. On the other hand, we will prove that each satisfying model for an $\mathcal{H} \mathcal{L}(\downarrow, E)$-formula $\varphi$ can be restricted to a submodel of doubly exponential size that still satisfies $\varphi$. This will allow for a guess-and-check procedure running in N2EXPTIME.

Lemma 6 For each $n \in \mathbb{N}$ there is a formula $\varphi_{n} \in \mathcal{H} \mathcal{L}(\downarrow$, E) with the following properties. 


\begin{tabular}{|c|c|c|c|c|c|c|}
\hline $\begin{array}{c}\text { values of } \\
C \quad D\end{array}$ & values of & values of & values of & & \multicolumn{2}{|c|}{ values of } \\
\hline $2^{n}-1 \quad \circ$ & $2^{n}-1 \quad \circ$ & $2^{n}-1 \bigcirc$ & $2^{n}-1 \bigcirc$ & & $2^{n}-1$ & $\leftarrow$ \\
\hline$\vdots$ & $\vdots$ & : & & & $\vdots$ & $\vdots$ \\
\hline 0 & 0 & 0 & 0 & $\ldots$ & 2 & - \\
\hline 10 & 0 & $1 \leftarrow$ & - & & 1 & $\leftarrow$ \\
\hline 0 & - & 0 & - & & 0 & $\leftarrow$ \\
\hline
\end{tabular}

Fig. 2 The behaviour of the counters $C$ and $D$ in an ER model

(i) $\left|\varphi_{n}\right| \in \mathcal{O}(n)$

(ii) $\varphi_{n} \in \mathcal{H} \mathcal{L}(\downarrow, E)-E R-S A T$

(iii) Each satisfying ER model for $\varphi_{n}$ has at least $2^{2^{n}}$ clusters with $2^{n}$ states each.

Proof In order to enforce a model of the required size, we will proceed in two steps. In the first step, we will implement a counter $C$ that ranges over the values $0, \ldots, 2^{n}-1$ within each cluster. This will make it possible, for each cluster, to distinguish $2^{n}$ states. The counter $C$ is realised by atomic propositions $c_{n-1}, \ldots, c_{0}$ whose truth values, in this order, constitute the binary representation of the value of $C$ at the respective state. (The "truth value" of $c_{i}$ at the state $m$ is 1 if $m \in V\left(c_{i}\right)$, and 0 otherwise, as usual.)

In the second step we will implement a counter $D$ that ranges over the values $0, \ldots, 2^{2^{n}}-1$ and distinguishes $2^{2^{n}}$ clusters (not states). It will be realised by one atomic proposition $d$. Given a cluster $X$, the binary representation of the value of $D$ at $X$ is determined by the truth values of $d$ at the states in $X$, in the order given by their $C$-values. Similar doubly exponential counters have been used, for instance, in Vardi and Stockmeyer (1985); Lange (2005) to establish lower bounds for propositional dynamic logics.

The required behaviour of $C$ and $D$ in a satisfying model for $\varphi_{n}$ is visualised in Fig. 2, where points and "sausages" represent states and clusters, respectively. The values of $C / D$ in each state/cluster are displayed next to it. In the case of $C$, the shown number determines the truth values of all $c_{i}$ as described above, and in case of $D$ the given number is the truth value of $d$. The respective value of the whole counter $D$ becomes readable after turning the $D$ column counterclockwise by 90 degrees. The state with $C=0$ in the cluster with $D=0$ shall be the state that satisfies $\varphi_{n}$. It is marked by a larger point.

All these enforcements, of course, will make heavy use of the $\downarrow$ operator combined with $\mathrm{E}$. We will now show how to achieve the required behaviour of $C$ and $D$. This will be via several formulae whose conjunction results in $\varphi_{n}$. We start with the conjuncts enforcing that each cluster has exactly $2^{n}$ states among which every value of $C$ between 0 and $2^{n}-1$ occurs once. In order to keep notation short, we will introduce some abbreviations. First, we would like to refer to specific $C$-values directly and abbreviate

$$
\operatorname{zero}(C)=\neg c_{0} \wedge \cdots \wedge \neg c_{n-1} \quad \text { and } \quad \operatorname{notmax}(C)=\neg c_{0} \vee \cdots \vee \neg c_{n-1}
$$

Second, it will be necessary to express that, for some $x \in \mathrm{SVAR}$, the $C$-value at the current state

(i) equals, 
(ii) equals one plus,

(iii) is less than,

(iv) is greater than

the $C$-value of the state to which $x$ is bound. We show how to express (ii) and (iii); the other two are easy or analogous. Recall that $@_{x} \psi$ abbreviates $\mathrm{E}(x \wedge \psi)$.

$$
\begin{aligned}
\operatorname{inc}\left(C_{x}\right)=\left(c_{0} \leftrightarrow @_{x} \neg c_{0}\right) \wedge \bigwedge_{k=1}^{n-1}\left[\left[\left(\neg c_{k-1} \wedge @_{x} c_{k-1}\right) \rightarrow\left(c_{k} \leftrightarrow @_{x} \neg c_{k}\right)\right]\right. \\
\left.\wedge\left[\neg\left(\neg c_{k-1} \wedge @_{x} c_{k-1}\right) \rightarrow\left(c_{k} \leftrightarrow @_{x} c_{k}\right)\right]\right] \\
\operatorname{less}\left(C_{x}\right)=\left(c_{n-1} \wedge @_{x} \neg c_{n-1}\right) \vee\left[( c _ { n - 1 } \leftrightarrow @ _ { x } c _ { n - 1 } ) \wedge \left[\left(c_{n-2}\right.\right.\right. \\
\left.\left.\left.\wedge @_{x} \neg c_{n-2}\right) \vee\left[\left(c_{n-2} \leftrightarrow @_{x} c_{n-2}\right) \wedge[\ldots]\right]\right]\right]
\end{aligned}
$$

The first macro says: "when going from the bits at $x$ to the bits at the current state, the least bit is flipped, and for every other bit it holds that: if the previous bit has changed from 1 to 0 , then this bit is flipped, otherwise it remains." The second macro says: "when going from the bits at $x$ to the bits at the current state, the highest bit has changed from 0 to 1 or [the highest bit remains and [the second highest bit has changed from 0 to 1 or [the second highest bit remains and [...]]]]." This formulation ensures that all macros are of linear size. The following conjuncts enforce the required behaviour of each cluster with respect to $C$.

- At the state satisfying $\varphi_{n}, C=0$ holds. zero $(C)$

- In each cluster there is a state with $C=0$. CZERO $=\mathrm{A} \diamond(\operatorname{zero}(C))$

- Each cluster has at most one state of each $C$-value. CUNIQUE $=\mathrm{A} \downarrow x$. (equal $\left(C_{x}\right) \rightarrow x$ )

- For each state of $C$-value $c<2^{n}-1$, there is a state of $C$-value $c+1$ in the same cluster.

$$
\text { CSUCC }=\mathrm{A}\left[\operatorname{notmax}(C) \rightarrow \downarrow x \cdot \diamond \operatorname{inc}\left(C_{x}\right)\right]
$$

We will now construct the part of $\varphi_{n}$ that implements the counter $D$. This requires expressing that the value of $D$ in the cluster of the current state equals one plus the value of $D$ in the cluster of the state assigned to some state variable $x$. The appropriate macro is described and illustrated in Fig. 3.

$$
\begin{aligned}
\operatorname{inc}\left(D_{x}\right)=\downarrow & y \cdot @_{x} \square \downarrow z \cdot\left[\left(\neg d \wedge \square\left(\operatorname{less}\left(C_{z}\right) \rightarrow d\right)\right) \rightarrow\right. \\
& {\left[@ _ { y } \square \left(\operatorname { e q u a l } ( C _ { z } ) \rightarrow \left(d \wedge \square\left(\operatorname{less}\left(C_{z}\right) \rightarrow \neg d\right)\right.\right.\right.} \\
& \left.\left.\left.\left.\wedge \square\left(\operatorname{greater}\left(C_{z}\right) \rightarrow \downarrow v \cdot @_{x} \square\left(\operatorname{equal}\left(C_{v}\right) \rightarrow\left(d \leftrightarrow @_{v} d\right)\right)\right)\right)\right)\right]\right]
\end{aligned}
$$

We easily obtain the two remaining conjuncts for $\varphi_{n}$.

- The state satisfying $\varphi_{n}$ belongs to a cluster with $D=0$. DZERO $=\square \neg d$

- For each cluster $X$ of $D$-value $d<2^{2^{n}}-1$, there is a cluster $Y$ of $D$-value $d+1$.

$$
\mathrm{DSUCC}=\mathrm{A} \downarrow x \cdot\left(\diamond \neg d \rightarrow \mathrm{E}\left(\operatorname{inc}\left(D_{x}\right)\right)\right)
$$




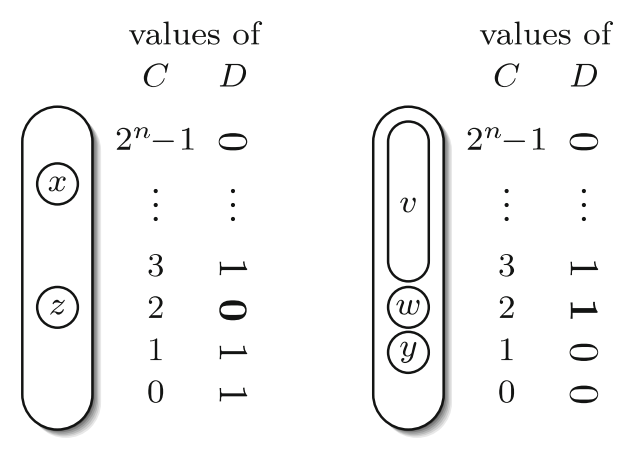

Fig. 3 Incrementation of the $D$ counter. Name the current state $y$. Name the state in the $x$-Cluster with $\neg d$ and lowest possible $C$-value $z$. For the state in the $y$-Cluster with the same $C$-value as $z$ (which we call $w$ only in this description and in the picture), require three things: (a) $d$ has to hold at $w$; (b) $\neg d$ has to hold at all states of the $y$-Cluster with $C$-value less than the $C$-value of $w$; (c) every state $v$ of the $y$-Cluster with $C$-value greater than the $C$-value of $w$ has to agree in $d$ with the states of the $x$-Cluster that have the same $C$-value as $v$.

Now let $\varphi_{n}=\operatorname{zero}(C) \wedge \mathrm{CZERO} \wedge \mathrm{CUNIQUE} \wedge \mathrm{CSUCC} \wedge \mathrm{DZERO} \wedge \mathrm{DSUCC}$. Since each of the above abbreviations is of linear size and they do not occur nested in $\varphi_{n}$, Part (i) of the theorem is satisfied. For (ii), it is easy to see that the following ER

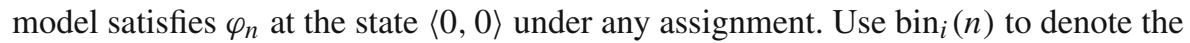
$i$-th bit in the binary representation of $n \in \mathbb{N}$, and let $\mathcal{M}^{n}=\left(M^{n}, R^{n}, V^{n}\right)$ as follows.

$$
\begin{array}{ll}
M^{n}=\{\langle x, y\rangle \mid x, y \in \mathbb{N} ; & \\
\left.0 \leqslant x<2^{2^{n}} ; 0 \leqslant y<2^{n}\right\} & V^{n}\left(c_{i}\right)=\left\{\langle x, y\rangle \mid \operatorname{bin}_{i}(y)=1\right\} \\
R^{n}=\left\{\left(\left\langle x_{1}, y_{1}\right\rangle,\left\langle x_{2}, y_{2}\right\rangle\right) \mid x_{1}=x_{2}\right\} & V^{n}(d)=\left\{\langle x, y\rangle \mid \operatorname{bin}_{y}(x)=1\right\}
\end{array}
$$

In order to show (iii), let $\mathcal{M}=(M, R, V)$ be an ER model with $m_{0,0} \in M$ and $g$ be an assignment for $\mathcal{M}$ such that $\mathcal{M}, g, m_{0,0} \Vdash \varphi_{n}$. Now the four $C$-conjuncts enforce that $C=0$ at $m_{0,0}$, and that each cluster of $\mathcal{M}$ contains exactly one state of $C$-value $c$ for each $c=0, \ldots, 2^{n}-1$. Due to DZERO, the $D$-value of $m_{0,0}$ 's cluster equals 0 , and DSUCC successively enforces the existence of a cluster of $D$-value $d$ for each $d=0, \ldots, 2^{2^{n}}-1$. (Note that the value of $D$ in each cluster is uniquely determined by $V(d)$ and the order of the cluster's states determined by their $C$-values.) Hence $\mathcal{M}$ has at least $2^{2^{n}}$ clusters with $2^{n}$ states each.

Corollary 1 For any function $f \in o(n),{ }^{3} \mathcal{H} \mathcal{L}(\downarrow, \mathrm{E})$ does not have the $2^{2^{f(n)}}$-size model property with respect to ER frames.

\section{Theorem $5 \mathcal{H} \mathcal{L}(\downarrow$, E)-ER-SAT is N2EXPTIME-hard.}

Proof We will reduce the $2^{2^{n}}$-tiling problem to $\mathcal{H} \mathcal{L}(\downarrow$, E)-ER-SAT. The reduction will use the techniques enforcing doubly exponentially large satisfying models from

\footnotetext{
3 The term $f \in o(n)$ means that the function $f$ is dominated by the identity function asymptotically (little-o notation).
} 


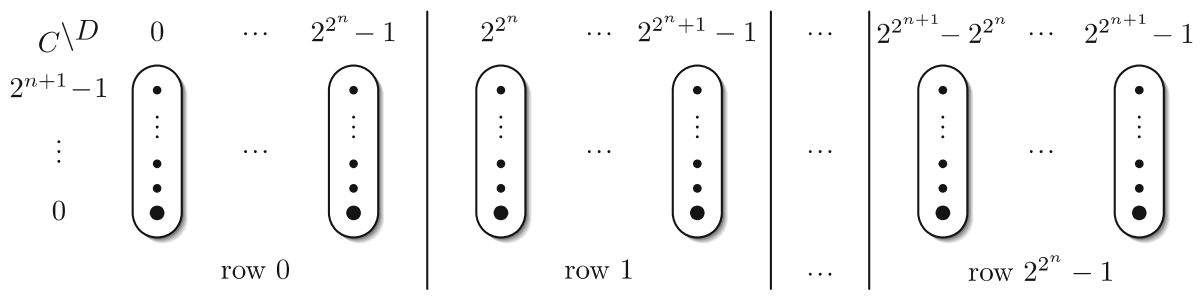

Fig. 4 Enforcing a tiling in an ER model of doubly exponential size

the proof of Lemma 6. In order to encode a tiling for the $2^{2^{n}} \times 2^{2^{n}}$-square in an ER model $\mathcal{M}$, we will first enforce that $\mathcal{M}$ has $2^{2^{n+1}}$ clusters with $2^{n+1}$ states each, using the same construction of counters $C$ and $D$, but with parameter $n+1$. The tiled square itself will be encoded in the states of $C$-value 0 of all clusters. Hence row 0 of the square will be in the clusters of $D$-value $0, \ldots, 2^{2^{n}}-1$; row 1 will be in the clusters of $D$-value $2^{2^{n}}, \ldots, 2 \cdot 2^{2^{n}}-1$; etc.; see Fig. 4 . The horizontal adjacencies in the original square can be expressed referring to pairs of clusters with successive $D$-values. In contrast, for the vertical adjacencies, pairs of clusters whose $D$-values differ by $2^{2^{n}}$ will have to be compared. ${ }^{4}$

For the required reduction, we will show how to transform an instance $\langle T, n\rangle$ of the tiling problem into a formula $\psi_{T, n}$ such that there is a $T$-tiling of the $2^{2^{n}} \times 2^{2^{n}}$-square if and only if $\psi_{T, n}$ is satisfiable. As in the proof of Lemma 6, this formula will consist of several conjuncts. The first of them will be the formula $\varphi_{n+1}$ from that proof, enforcing the required structure of the model. In order to keep the remaining conjuncts short, we will use the same abbreviations again, but with $n+1$ instead of $n$. Furthermore, $\operatorname{inc}\left(D_{x}, 2^{2^{n}}\right)$ denotes that the $D$-value of the current state's cluster equals $2^{2^{n}}$ plus the $D$-value of the cluster containing the state to which $x$ is bound. This abbreviation is defined analogously to the shortcut inc $\left(D_{x}\right)$.

Now we are ready to give the conjuncts that enforce the tiling. Let $T$ be a set of tile types. For each tile type $t$ we will use an atomic proposition $t$ to denote that a tile of type $t$ lies at the respective position.

- At each state with $C$-value 0 lies exactly one tile.

$$
\operatorname{TILE}=\mathrm{A}\left(\operatorname{zero}(C) \rightarrow \bigvee_{t \in T}\left(t \wedge \bigwedge_{\substack{t^{\prime} \in T \\ t^{\prime} \neq t}} \neg t\right)\right)
$$

- Tiles match horizontally and vertically. (The $\diamond$-subformulae require that corresponding position of the current state does not belong to the last column (or row,

\footnotetext{
4 Note that this is not the standard way to encode tilings in models, insofar as not every state of the model corresponds to a position in the grid. However, this modification of the standard way is not new, since it relies on ideas developed by Chlebus (1986) to encode the rectangle tiling problem with exponential parameter into a more intricate version of a bounded tiling problem that he called "High Tiling".
} 
respectively) of the square.)

$$
\begin{aligned}
& \mathrm{HOR}=\mathrm{A}\left[( \operatorname { z e r o } ( C ) \wedge \diamond ( \neg c _ { n } \wedge d ) ) \rightarrow \downarrow x \cdot \left(\bigwedge_{t \in T} t \rightarrow \mathrm{A}((\operatorname{zero}(C)\right.\right. \\
&\left.\left.\left.\left.\qquad \operatorname{inc}\left(D_{x}\right)\right) \rightarrow \bigvee_{t^{\prime} \in \mathrm{RI}(t)} t^{\prime}\right)\right)\right] \\
& \operatorname{VER}=\mathrm{A}\left[( \operatorname { z e r o } ( C ) \wedge \diamond ( c _ { n } \wedge \neg d ) ) \rightarrow \downarrow x \cdot \left(\bigwedge_{t \in T} t \rightarrow \mathrm{A}((\operatorname{zero}(C)\right.\right. \\
&\left.\left.\left.\left.\wedge \operatorname{inc}\left(D_{x}, 2^{2^{n}}\right)\right) \rightarrow \bigvee_{t^{\prime} \in \mathrm{UP}(t)} t^{\prime}\right)\right)\right]
\end{aligned}
$$

- The borders of the square are white.

$$
\begin{aligned}
& \text { WHITE }=\mathrm{A}\left[\left(\square\left(c_{n} \rightarrow \neg d\right) \rightarrow \bigvee_{\substack{t \in T \\
\operatorname{bot}(t)=\text { white }}} t\right) \wedge\left(\square\left(c_{n} \rightarrow d\right) \rightarrow \bigvee_{\substack{t \in T \\
\operatorname{top}(t)=\text { whits }}} t\right)\right. \\
& \left.\wedge\left(\square\left(\neg c_{n} \rightarrow \neg d\right) \rightarrow \bigvee_{\substack{t \in T \\
\text { left }(t)=\text { white }}} t\right) \wedge\left(\square\left(\neg c_{n} \rightarrow d\right) \rightarrow \bigvee_{\substack{t \in T \\
\operatorname{right}(t)=\text { white }}} t\right)\right]
\end{aligned}
$$

Now let $\psi_{T, n}=\varphi_{n+1} \wedge$ TILE $\wedge$ HOR $\wedge$ VER $\wedge$ WHITE. Each conjunct is of size at most $\mathcal{O}\left(n^{2}+|T|^{2}\right)$. From their definitions it is clear that $\psi_{T, n}$ can be computed in time polynomial in $n+|T|$. It remains to show that there is a $T$-tiling of the $2^{2^{n}} \times 2^{2^{n}}$-square if and only if $\psi_{T, n} \in \mathcal{H} \mathcal{L}(\downarrow$, E)-ER-SAT.

“ $\Rightarrow$ ”. Suppose there is a tiling $\tau$ for the $2^{2^{n}} \times 2^{2^{n}}$ square. We use this tiling to construct a model $\mathcal{M}^{n+1}=\left(M^{n+1}, R^{n+1}, \bar{V}^{n+1}\right)$ for $\psi_{T, n}$, where $M^{n+1}$ and $R^{n+1}$ are given as in the proof of Lemma 6(ii), and $\bar{V}^{n+1}$ is $V^{n+1}$ from (3) plus

$$
V(t)=\left\{\left\langle 2^{2^{n}} \cdot i+j, 0\right\rangle \mid \tau(i, j)=t\right\}, \quad \text { for } t \in T .
$$

Now it is easy to see that $\mathcal{M}, g,\langle 0,0\rangle \Vdash \psi_{T, n}$ for any assignment $g$ : The first conjunct, $\varphi_{n+1}$, is treated in the proof of Lemma 6(ii). The remaining conjuncts hold at $\langle 0,0\rangle$ due to the definition of $V$, the fact that $\tau$ is a function, and the tiling conditions.

“Æ”. $\quad$ Suppose $\psi_{T, n} \in \mathcal{H} \mathcal{L}(\downarrow$, E)-ER-SAT. Then there exist a model $\mathcal{M}=(M, R$, $V$ ), an assignment $g$ for $\mathcal{M}$, and a state $m_{0,0} \in M$ such that $\mathcal{M}, g, m_{0,0} \Vdash \psi_{T, n}$. Because of the conjunct $\varphi_{n+1}$ of $\psi_{T, n}$, consulting the proof of Lemma 6(iii) shows that for every $x<2^{2^{n+1}}$ and every $y<2^{n+1}$, there are clusters $\mathrm{Cl}_{x}$ with states 
$m_{x, y} \in \mathrm{Cl}_{x}$ such that $C$ has value $y$ in each $m_{x, y}$, and $D$ has value $x$ in each $\mathrm{Cl}_{x}$. This allows for constructing a tiling $\tau$ from the states $m_{x, 0}$ via

$$
\tau(i, j)=t \Leftrightarrow m_{x, 0} \in V(t) \quad\left(\text { for } x=2^{2^{n}} \cdot i+j\right)
$$

The correctness of this definition is ensured by the conjunct TILE. Due to the remaining conjuncts, $\tau$ defines a permissible tiling.

We will now establish the corresponding upper bound, showing that the full hybrid language has a doubly exponential size model property over ER frames. This will make it possible to decide satisfiability using a straightforward check-and-guess procedure and involving results for model checking.

Lemma $7 \mathcal{H} \mathcal{L}(\downarrow, E)$ has the $2^{2^{2 n+2}}$-size model property with respect to ER frames.

Proof Intuitively, the proof relies on the following considerations: Call the set of propositional variables and nominals that hold at a given state of a model the type of this state. Let the $C$-type of a cluster be the set of types of all points of this cluster. If we had no $\downarrow$ in our language, then two states of the same type that belong to the same cluster would not be distinguishable, that is, they would satisfy the same formulae. Even two states of the same type that belong to two different clusters of the same C-type would not be distinguishable. This would enable us to restrict clusters to at most one state per possible type and to restrict a whole satisfying model for some formula $\varphi$ to at most one cluster per possible C-type without affecting satisfiability of $\varphi$. In the presence of $\downarrow$, this argumentation must be refined and requires a certain amount of technical details. Let $\varphi$ be a formula of size $n$ and $\mathcal{M}=(M, R, V)$ be a satisfying model for $\varphi$. First, there are at most $2^{n}$ possible types of states. Since an assignment for $\mathcal{M}$ might bind all state variables occurring in $\varphi$ to different states of the same type, only up to $n+1$ states of the same type belonging to the same cluster are distinguishable. Hence, it is legitimate to restrict each cluster of $\mathcal{M}$ to at most $n+1$ states of each type in the first step, which leads to an exponential bound in the size of clusters.

In the second step, we modify the notion of a C-type of a cluster $X$ to be the multiset containing as many copies of each type as there are states of this type in $X$, but not more than $n+1$. It is legitimate, too, to restrict the whole model to at most $n+1$ clusters of each C-type. Since there are at most $(n+2)^{2^{n}}$ many different C-types, the number of clusters - and, hence, states - of the restricted model is bounded by $2^{2^{\mathcal{O}(n)}}$. The formal proof of the $2^{2^{2 n+2}}$-size model property requires quite some notation. Let $\varphi \in \mathcal{H} \mathcal{L}(\downarrow, \mathrm{E})$-ER-SAT be of size $n$. Then there exist an ER model $\mathcal{M}=(M, R, V)$, an assignment $g_{0}$ for $\mathcal{M}$, and a state $m_{0} \in \mathcal{M}$ such that $\mathcal{M}, g_{0}, m_{0} \Vdash \varphi$. Let $C_{i} \subseteq M, i \in I$, be all clusters of $\mathcal{M}$, for an appropriate index set $I$ that contains 0 , such that $m_{0} \in C_{0}$. Let $x_{1}, \ldots, x_{s}$ be all state variables occurring in $\varphi$. Analogously, let $a_{1}, \ldots, a_{t}$ be all other atoms in $\varphi$. Clearly $s, t \leqslant n$. A $\varphi$-type is a subset of $\left\{a_{1}, \ldots, a_{t}\right\}$. Let $A_{1}, \ldots, A_{2^{t}}$ be an enumeration of all $\varphi$-types, such that $m_{0}$ is of type $A_{1}$. (A state $m$ is of type $A_{\ell}$ iff for each $j=1, \ldots, t$ : ( $\left.m \in V\left(a_{j}\right) \Leftrightarrow a_{j} \in A_{\ell}\right)$. Furthermore, we will deliberately speak of "(C-)types" 
Fig. 5 Dividing a cluster into "type layers"

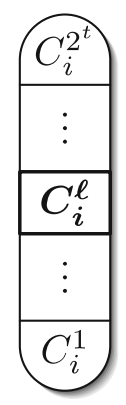

instead of " $\varphi$-(C-)types" whenever no confusion may arise.) Given a cluster $C$, we divide it into $2^{t}$ "type layers" $C_{i}^{\ell}=\left\{m \in C_{i} \mid m\right.$ is of type $\left.A_{\ell}\right\}$, as shown in Fig. 5 .

We define a function $f: I \times\left\{1, \ldots, 2^{t}\right\} \rightarrow \mathfrak{P}(M)$ that assigns a set of states to each pair $\langle i, \ell\rangle$ of a cluster number $i$ and a type number $\ell$, such that $f(i, \ell)$ is a subset of $C_{i}$. The union of all possible $f(i, \ell)$ will constitute the first restriction of $\mathcal{M}$. The function $f$ is defined as follows, where $\# C_{i}^{\ell}$ denotes the number of states in $C_{i}^{\ell}$. If $\# C_{i}^{\ell} \leqslant s+1$, then $f(i, \ell)=C_{i}^{\ell}$. Otherwise, $f(i, \ell)$ is some subset of $C_{i}^{\ell}$ of size at most $s+1$ that satisfies the following conditions.

(i) For each $j=1, \ldots, s$ : if $g_{0}\left(x_{j}\right) \in C_{i}^{\ell}$, then $g_{0}\left(x_{j}\right) \in f(i, \ell)$.

(ii) $m_{0} \in f(0,1)$.

Such a subset always exists. For any cluster $C_{i}$, let $f\left(C_{i}\right)$ denote the union of all $f(i, \ell)$. Due to the definition of $f, f\left(C_{i}\right) \subseteq C_{i}$, and $f\left(C_{i}\right)$ has at most $(s+1) \cdot 2^{t}$ states. We denote the union of all $f\left(C_{i}\right)$ by $M^{\prime}$.

After restricting the cluster size, we will restrict the number of the clusters. Let $\mathcal{A}$ be the multiset containing $s+1$ copies of each type $A_{\ell}$. Call each subset of $\mathcal{A}$ a $\varphi$-C-type. The power set $\mathfrak{P}(\mathcal{A})$ contains $(s+2)^{2^{t}}$ elements. Let $\mathcal{A}_{1}, \ldots, \mathcal{A}_{(s+2)^{2^{t}}}$ be an enumeration of all $\varphi$-C-types, such that $f\left(C_{0}\right)$ is of C-type $\mathcal{A}_{1}$. (The C-type of a cluster $C_{i}$ is determined by the number of states of each type in its restriction $f\left(C_{i}\right)$.) We divide $M^{\prime}$ into $(s+2)^{2^{t}}$ "C-type layers" $\mathcal{C}_{\ell}$ being the union of $f\left(C_{i}\right)$ for all $C_{i}$ of C-type $\mathcal{A}_{\ell}$.

Now define a second choice function $f^{\prime}:\left\{1, \ldots,(s+2)^{2^{t}}\right\} \rightarrow \mathfrak{P}\left(M^{\prime}\right)$ that assigns a set of states to each C-type number such that $f^{\prime}(\ell)$ is a union of (restricted) clusters. The union of all possible $f^{\prime}(\ell)$ will constitute the second restriction of $\mathcal{M}$. The function $f^{\prime}$ is defined as follows. If there are not more than $s+1$ clusters of C-type $\mathcal{A}_{\ell}$, then $f^{\prime}(\ell)=\mathcal{C}_{\ell}$. Otherwise, $f^{\prime}(\ell)$ is the union of $s+1$ restricted clusters of type $\mathcal{A}_{\ell}$ satisfying

(iii) $\forall j=1, \ldots, s$ : if $g_{0}\left(x_{j}\right) \in f\left(C_{i}\right)$ for some $C_{i}$ of type $\mathcal{A}_{\ell}$, then $f\left(C_{i}\right) \subseteq f^{\prime}(\ell)$; (iv) $f\left(C_{0}\right) \subseteq f^{\prime}(1)$.

Such a subset always exists. Due to the definition of $f^{\prime}$, each $f^{\prime}(\ell)$ contains at most $s+1$ restricted clusters and, hence, $(s+1)^{2} \cdot 2^{t}$ states. We now construct a new model $\mathcal{M}^{\prime \prime}=\left(M^{\prime \prime}, R^{\prime \prime}, V^{\prime \prime}\right)$ from $\mathcal{M}$, where $M^{\prime \prime}$ is the union of $f^{\prime}(\ell)$ for all C-types $\mathcal{A}_{\ell}$, and $R^{\prime \prime}$ and $V^{\prime \prime}$ are the restrictions of $R$ and $V$ to $M^{\prime \prime}$. Now the following facts about $\mathcal{M}^{\prime \prime}$ are obvious. It is still an ER model, whose clusters are restrictions of clusters of $\mathcal{M}$. 
It contains $m_{0}$, because $m_{0} \in f\left(C_{0}\right) \subseteq f^{\prime}(1)$. The assignment $g_{0}$ is an assignment for $\mathcal{M}^{\prime \prime}$. Since there are $(s+2)^{2^{t}}$ C-types, $M^{\prime \prime}$ contains $(s+2)^{2^{t}} \cdot(s+1)^{2} \cdot 2^{t}$ states. This number is limited by $2^{2^{2 n+2}}$ because $s, t \leqslant n$.

It remains to show that $\mathcal{M}^{\prime \prime}, g_{0}, m_{0} \Vdash \varphi$. For this purpose, we make use of an auxiliary statement. This statement uses the concept of agreement in a pair of assignments. We say that two states $m$ and $m^{\prime}$ from $\mathcal{M}$ agree in two assignments $g / g^{\prime}$ for $\mathcal{M}$ iff $\left\{x_{k} \mid g\left(x_{k}\right)=m\right\}=\left\{x_{k} \mid g^{\prime}\left(x_{k}\right)=m^{\prime}\right\}$. Two clusters $C_{i}$ and $C_{i^{\prime}}$ agree in $g / g^{\prime}$ iff they are of the same C-type, and for each $A_{\ell}$, each $m \in C_{i}^{\ell}$, there is some $m^{\prime} \in C_{i^{\prime}}^{\ell}$ that agrees with $m$ in $g / g^{\prime}$.

Claim For each subformula $\psi$ of $\varphi$; for each two assignments $g, g^{\prime}$ for $\mathcal{M}$; for each C-type $\mathcal{A}_{\ell}$; for each two clusters $C_{i}$ and $C_{i^{\prime}}$ that agree in $g / g^{\prime}$; for each type $A_{\ell}$; and for each $m \in C_{i}^{\ell}$ and $m^{\prime} \in C_{i^{\prime}}^{\ell}$ that agree in $g / g^{\prime}$; it holds that $\mathcal{M}, g, m \Vdash \psi$ iff $\mathcal{M}, g^{\prime}, m^{\prime} \Vdash \psi$.

Proof of Claim By induction on $\psi$. Direction “ $\Rightarrow$ " suffices because of the symmetry of the conditions on $m$ and $m^{\prime}$. The atomic and Boolean cases of the induction are immediate and easy, respectively. The E case is trivial, and the $\downarrow$ case is straightforward if one considers the fact that, since $m$ and $m^{\prime}$ agree in $g / g^{\prime}$, they also agree in $g_{m}^{x} /\left(g^{\prime}\right)_{m^{\prime}}^{x}$, for any state variable $x$. The only interesting case is the $\diamond$ case, with the following argumentation. Suppose $\mathcal{M}, g, m \Vdash \diamond \vartheta$. Then there exists some $\bar{m} \in C_{i}$ with $\mathcal{M}, g, \bar{m} \Vdash \vartheta$. Let $A_{\ell^{\prime}}$ be the $\varphi$-type of $\bar{m}$. Then $C_{i}^{\ell^{\prime}}$ and, hence, $C_{i^{\prime}}^{\ell^{\prime}}$ is not empty. Because $C_{i}$ and $C_{i^{\prime}}$ agree in $g / g^{\prime}$, there is some $\bar{m}^{\prime} \in C_{i^{\prime}}^{\ell^{\prime}}$ that agrees with $\bar{m}$ in $g^{\prime} / g$. Due to the induction hypothesis, $\mathcal{M}, g^{\prime}, \bar{m}^{\prime} \Vdash \vartheta$. Hence, $\mathcal{M}, g^{\prime}, m^{\prime} \Vdash \diamond \vartheta$.

Now the required fact $\mathcal{M}^{\prime \prime}, g_{0}, m_{0} \Vdash \varphi$ is a consequence of the following claim.

Claim For each subformula $\psi$ of $\varphi$, for each $m \in M^{\prime \prime}$, for each assignment $g$ for $\mathcal{M}$ ", it holds that $\mathcal{M}, g, m \Vdash \psi$ iff $\mathcal{M}^{\prime \prime}, g, m \Vdash \psi$.

Proof of Claim Since $m \in M^{\prime \prime}$, there is some $i \in I$ such that $m \in f\left(C_{i}\right) \subseteq M^{\prime \prime}$. Let $\mathcal{A}_{\ell}$ be the C-type of $C_{i}$. We prove the claim by induction. The atomic cases follow from the facts that $\mathcal{M}^{\prime \prime}$ is a restriction of $\mathcal{M}$ and that $g$ is an assignment for both $\mathcal{M}$ and $\mathcal{M}^{\prime \prime}$. The boolean cases are straightforward. So is the $\downarrow$ case if one considers the fact that $g_{m}^{x}$ is still an assignment for $\mathcal{M}^{\prime \prime}$. For the remaining cases for $\diamond$ and $\mathrm{E}$, the " $\Leftarrow$ " direction is trivial. We will only prove the " $\Rightarrow$ " direction.

Case $\psi=\diamond \vartheta$. Suppose $\mathcal{M}, g, m \Vdash \diamond \vartheta$. Then there exists some $m^{\prime} \in C_{i}$ with $\mathcal{M}, g, m^{\prime} \Vdash \vartheta$. Let the type of $m^{\prime}$ be $A_{k}$. There are three cases to distinguish.

(1) $\# C_{i}^{k} \leqslant s+1$. Then $m^{\prime}$ belongs to $f(i, k)$ and, hence, to $f\left(C_{i}\right)$. Hence $m^{\prime} \in M^{\prime \prime}$ and $m R^{\prime \prime} m^{\prime}$. Together with the induction hypothesis, this immediately yields $\mathcal{M}^{\prime \prime}, g, m \Vdash \diamond \vartheta$.

(2) $\# C_{i}^{k}>s+1$ and, for some $j=1, \ldots, s, g\left(x_{j}\right)=m^{\prime}$. Since $g$ is for $\mathcal{M}^{\prime \prime}$, we obtain $m^{\prime} \in M^{\prime \prime}$ and $m R^{\prime \prime} m^{\prime}$, which yields $\mathcal{M}^{\prime \prime}, g, m \Vdash \diamond \vartheta$ as in case (1).

(3) $\# C_{i}^{k}>s+1$ and, for no $j=1, \ldots, s, g\left(x_{j}\right)=m^{\prime}$. Due to the size of $C_{i}^{k}$ and the construction of $f$, there is some $m^{\prime \prime} \in f(i, k)$ not affected by $g$ either. Since $m^{\prime}$ and $m^{\prime \prime}$ are of the same type and agree in $g / g$, the previous claim implies that $\mathcal{M}, g, m^{\prime \prime} \Vdash \vartheta$. The remaining argumentation is the same as in case (1), with $m^{\prime \prime}$ instead of $m^{\prime}$. 
Case $\psi=\mathrm{E} \vartheta$. Suppose $\mathcal{M}, g, m \Vdash \mathrm{E} \vartheta$. Then there exists some $m^{\prime} \in M$ with $\mathcal{M}, g, m^{\prime} \Vdash \vartheta$. Let the type of $m^{\prime}$ be $A_{k}$, and let $m^{\prime}$ be from $C_{i^{\prime}}$, the latter being of C-type $\mathcal{A}_{\ell}$. As in the $\diamond$ case, there are three sub-cases to distinguish.

(1) There are at most $s+1$ clusters of C-type $\mathcal{A}_{\ell}$. Then $C_{i}$ and, hence, $m^{\prime}$ belong to $\mathcal{M}^{\prime \prime}$. Together with the induction hypothesis, this immediately yields $\mathcal{M}^{\prime \prime}, g, m \Vdash$ $\mathrm{E} \vartheta$.

(2) There are more than $s+1$ clusters of C-type $\mathcal{A}_{\ell}$ and, for some $j=1, \ldots, s$, $g\left(x_{j}\right) \in f\left(C_{i^{\prime}}\right)$. Since $g$ is for $\mathcal{M}^{\prime \prime}$, we obtain $f\left(C_{i^{\prime}}\right) \subseteq M^{\prime \prime}$, which yields $\mathcal{M}^{\prime \prime}, g, m \Vdash \mathrm{E} \vartheta$ as in case (1).

(3) There are more than $s+1$ clusters of C-type $\mathcal{A}_{\ell}$ and, for no $j=1, \ldots, s$, $g\left(x_{j}\right) \in f\left(C_{i^{\prime}}\right)$. Due to the "large enough" number of clusters of C-type $\mathcal{A}_{\ell}$ and the construction of $f^{\prime}$, there is some cluster $C_{i^{\prime \prime}} \subseteq f^{\prime}(\ell)$ not affected by $g$ either. Since $C_{i^{\prime}}$ and $C_{i^{\prime \prime}}$ agree in $g / g$, there is some $m^{\prime \prime} \in f\left(C_{i^{\prime \prime}}\right)$, having the same type as $m^{\prime}$ and agreeing with $m^{\prime}$ in $g / g$. Hence, due to the previous claim, $\mathcal{M}, g, m^{\prime \prime} \Vdash \vartheta$. Here we have to distinguish the same three sub-cases as in the $\diamond$ case. The argumentation is analogous and leads to the required result $\mathcal{M}^{\prime \prime}, g, m \Vdash \mathrm{E} \vartheta$.

\section{Theorem $6 \mathcal{H} \mathcal{L}(\downarrow, \mathrm{E})$-ER-SAT is N2EXPTIME-complete.}

Proof The lower bound follows from Theorem 5. For the upper bound, let $\varphi$ be an arbitrary instance of $\mathcal{H L}(\downarrow, E)$-ER-SAT. In order to determine whether $\varphi \in \mathcal{H} \mathcal{L}(\downarrow, \mathrm{E})$-ER-SAT, we guess a model $\mathcal{M}=(M, R, V)$, an assignment $g$, and a state $m \in M$, and check whether $\mathcal{M}, g, w \Vdash \varphi$. Let $n=|\varphi|$. If $\varphi \in \mathcal{H} \mathcal{L}(\downarrow$, E)-ER-SAT, then, due to Lemma 7, it has a satisfying model with state space $M$ of size at most $2^{2^{2 n+2}}$. Hence, in time $\mathcal{O}\left(2^{2^{2 n+2}}\right)$ we can guess a model $\mathcal{M}=(M, R, V)$ of size at most $2^{2^{2 n+2}}$ and check whether $R$ is an equivalence relation. An assignment $g$ can be guessed in time $\mathcal{O}\left(2^{3 n+2}\right)$. All the guesses together take time $\mathcal{O}\left(2^{2^{k^{\prime} \cdot n}}\right)$ for a constant $k^{\prime}$.

Finally, checking whether $\mathcal{M}, g \Vdash \varphi$ can be done using procedure MCFULL from Franceschet and de Rijke (2005). By (Franceschet and de Rijke, 2005, Theorem 4.5) this takes time $\mathcal{O}\left(|\varphi| \cdot(|M|+|R|) \cdot|M|^{k}\right)=\mathcal{O}\left(n \cdot\left(2^{2^{2 n+2}}+\left(2^{2^{2 n+2}}\right)^{2}\right) \cdot\left(2^{2^{2 n+2}}\right)^{k}\right)=$ $\mathcal{O}\left(2^{2^{k^{\prime \prime} \cdot n}}\right)$ for an appropriate constant $k^{\prime \prime}$. Altogether, we have a nondeterministic algorithm that runs in doubly exponential time.

\subsection{Pure languages with binders}

Satisfiability for all pure languages with binders is PSPACE-complete. The lower bound follows almost immediately from Lemma 1 . The upper bound uses a polynomial-size model property that is obtained in a similar manner as the $2^{2^{2 n+2}}$-size model property for $\mathcal{H} \mathcal{L}(\downarrow, E)$ in Lemma 7. Note the following subtle difference in argumentation. While the $2^{2^{2 n+2}}$-size model property of $\mathcal{H} \mathcal{L}(\downarrow, E)$ implies an N2EXPTiME upper bound for satisfiability, the polynomial-size model property of a binder language does not imply an NP upper bound for satisfiability. The reason becomes clear 
if we recall the general complexity results for model checking over arbitrary frames from Franceschet and de Rijke (2005): In the presence of binders, this problem is PSPACE-complete, but an upper time bound is $\mathcal{O}\left(|\varphi| \cdot|M|^{2|\varphi|}\right)$. If the model is large compared to the formula, as in the case of $\mathcal{H} \mathcal{L}(\downarrow, E)$, then the factor $|\varphi|$ in the exponent is unimportant. In the case of a polynomial-size model property, however, the upper time bound for model checking only yields an exponential time bound for the whole guess-and-check algorithm deciding satisfiability.

Theorem 7 Let $X$ be $\{\downarrow\},\{\downarrow, @\},\{\exists\}$, or $\{\downarrow$, E $\}$. Then $\mathcal{P} \mathcal{H} \mathcal{L}(X)$-compl-SAT and $\mathcal{P H} \mathcal{L}(X)$-ER-SAT are PSPACE-complete.

Proof PSPACE-hardness follows from Lemma 1 and the fact that the model $\mathcal{M}$ used in that proof can be axiomatised using $\square(t \vee f)$. This allows to extend the reduction from QSAT to $\mathcal{P} \mathcal{H} \mathcal{L}(\downarrow)$-compl-SAT.

Containment in PSPACE follows from the fact that $\mathcal{P} \mathcal{H} \mathcal{L}(\downarrow, \mathrm{E})$ has the $\mathcal{O}\left(n^{2}\right)$-size model property with respect to ER frames. The proof of this property is analogous to the proof of Lemma 7, but with one fundamental difference. Since our language is pure, the number of types decreases to one. Hence, in each cluster, at most $n+1$ different states can be distinguished by means of state variables. This means that there are only $n+1$ C-types (representing clusters with $1,2, \ldots, n+1$ states), and, again, only $n+1$ clusters of each C-type can be distinguished. This leads to a $(n+1)^{2}$-size model property. The technical details are essentially the same as in the proof of Lemma 7.

Now a model can be guessed and checked in polynomial space (Theorem 1). Since PSPACE is closed under nondeterminism, the upper bound follows.

\section{Conclusion}

We have completely classified the computational complexity of model checking and satisfiability over ER frames and complete frames for all hybrid languages shown in Fig. 1a. In detail, we have established the following results.

Model checking over both frame classes is in polynomial time for each binder-free language, and PSPACE-complete in the cases with binders. In all seven cases, the pure fragment has the same complexity.

Satisfiability over both frame classes is NP-complete for all binder-free cases, whether pure or with propositional variables. This is the same complexity as for modal logic over equivalence relations (Ladner 1977). For the four languages with binders, there is a significant gap in complexity between the pure and non-pure cases. The former are PSPACE-complete, while the latter are NEXPTIME-complete with one exception: The full language with $\mathrm{E}$ and $\downarrow$ is N2EXPTIME-complete, but only over ER frames. For this case, we have established a $2^{2^{2 n+2}}$-size model property for $\mathcal{H} \mathcal{L}(\downarrow, \mathrm{E})$ with respect to ER frames, and we have disproven a $2^{2^{o(n)}}$-size model property.

The scope of our results is slightly larger than stated in Theorems 1, 3, 6, and 7, in the sense that all these statements hold as well for the nominal-free fragments of all sentences of the respective languages $\mathcal{H} \mathcal{L}(\cdot)$ and $\mathcal{P} \mathcal{H} \mathcal{L}(\cdot)$. This is due to the fact that neither nominals nor free state variables occur in the particular reductions used for the 
lower bounds. (Except for the case of the use of (Mundhenk et al., 2005, Theorem 4), to be precise. However, the lower NEXPTIME bound for $\mathcal{H} \mathcal{L}(\downarrow)$-compl-SAT does hold for nominal-free sentences as well, because nominals and free state variables can be simulated in complete frames using bound state variables.) The only case in which the lower bound does not carry over to the pure fragment is that of satisfiability for binder-free languages (see Theorem 2).

Acknowledgements The authors thank the referee and Ulrike Sattler for helpful comments and suggestions, and for an improvement to the proof of Lemma 6 .

\section{References}

Areces, C., Blackburn, P., \& Marx, M. (1999). A road-map on complexity for hybrid logics. In Proceedings of the 13th CSL, 1999, volume 1683 of LNCS (pp. 307-321). Springer.

Areces, C., Blackburn, P., \& Marx, M. (2000). The computational complexity of hybrid temporal logics. Logic Journal of the IGPL, 8(5), 653-679.

Blackburn, P., \& Seligman, J. (1995). Hybrid languages. Journal of Logic, Language and Information, 4, 251-272.

Blackburn, P. \& Seligman, J. (1998). What are hybrid languages? In M. Kracht, M. de Rijke, H. Wansing, \& M. Zakharyaschev (Eds.), Advances in Modal Logic, volume 1 of CSLI Publications (pp. 41-62). Stanford University.

Chlebus, B. S. (1986). Domino-tiling games. Journal of Computer and System Sciences, 32(3), 374-392.

Franceschet, M. \& de Rijke, M. (2005). Model checking for hybrid logics (with an application to semistructured data). Journal of Applied Logic, 4(3), 279-304.

Franceschet, M., de Rijke, M., \& Schlingloff, B.-H. (2003). Hybrid logics on linear structures: Expressivity and complexity. In Proceedings of the 10th TIME, 2003 (pp. 166-173). IEEE Computer Society.

Goranko, V. (1996). Hierarchies of modal and temporal logics with reference pointers. Journal of Logic, Language and Information, 5(1), 1-24.

Ladner, R. E. (1977). The computational complexity of provability in systems of modal propositional logic. SIAM Journal on Computing, 6(3), 467-480.

Lange, M. (2005). A lower complexity bound for propositional dynamic logic with intersection (Vol. 5, pp. 133-147). King's College Publications.

Mundhenk, M., Schneider, T., Schwentick, T., \& Weber, V. (2005). Complexity of hybrid logics over transitive frames. In H. Schlingloff (Ed.), M4M, volume 194 of Informatik-Berichte (pp. 62-78). Humboldt-Universität zu Berlin. The cited version is http://arxiv.org/abs/0806.4130v1atarXiv.org.

Papadimitriou, C. H. (1994). Computational complexity. Addison-Wesley.

Savelsbergh, M. \& van Emde Boas, P. (1984). Bounded tiling, an alternative to satisfiability. In G. Wechsung (Ed.), 2nd Frege Conference, volume 20 of Mathematische Forschung (pp. 354-363). Berlin: Akademie Verlag.

ten Cate, B., \& Franceschet, M. (2005). On the complexity of hybrid logics with binders. In Proceedings of the 19th CSL, 2005, volume 3634 of LNCS (pp. 339-354). Springer.

Vardi, M., \& Stockmeyer, L. (1985). Improved upper and lower bounds for modal logics of programs. In Proceedings of the 17th STOC (pp. 240-251). 